\title{
Lincoln à Cooper Union : Plaidoyer pour une critique néo-classique
}

Lincoln at Cooper Union: a Rationale for Neo-Classical Criticism

Michael Leff et Gerald P. Mohrmann

Traducteur : Sivan Cohen-Wiesenfeld

\section{(C) OpenEdition}

1 Journals

Édition électronique

URL : http://journals.openedition.org/aad/1080

DOI : $10.4000 /$ aad. 1080

ISSN : 1565-8961

Éditeur

Université de Tel-Aviv

Référence électronique

Michael Leff et Gerald P. Mohrmann, «Lincoln à Cooper Union : Plaidoyer pour une critique néoclassique », Argumentation et Analyse du Discours [En ligne], 6 | 2011, mis en ligne le 15 avril 2011, consulté le 23 septembre 2019. URL : http://journals.openedition.org/aad/1080 ; DOI : 10.4000/aad. 1080

Ce document a été généré automatiquement le 23 septembre 2019.

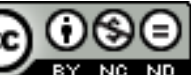

Argumentation \& analyse du discours est mis à disposition selon les termes de la licence Creative Commons Attribution - Pas d'Utilisation Commerciale - Pas de Modification 4.0 International. 


\title{
Lincoln à Cooper Union : Plaidoyer pour une critique néo-classique
}

\author{
Lincoln at Cooper Union: a Rationale for Neo-Classical Criticism \\ Michael Leff et Gerald P. Mohrmann \\ Traduction : Sivan Cohen-Wiesenfeld
}

\section{NOTE DE L'ÉDITEUR}

Cet article a paru en anglais dans Quarterly Journal of Speech 1974, $60: 4,459-467$, et est reproduit avec l'autorisation de la revue.

1 Le dernier numéro du Quarterly Journal of Speech présentait notre analyse critique du discours de Lincoln à Cooper Union. Bien que les considérations méthodologiques y soient peu développées, nous supposons que les fondements néo-classiques en sont apparents. Nous avions proposé une explication plus élaborée de notre approche méthodologique dans une première version; mais les éditeurs avaient estimé qu'un unique article n'offrait pas assez d'espace pour tout à la fois justifier la démarche, et en faire une application concrète. Nous en remettant à cet avis, nous avons presque entièrement supprimé la partie théorique. Après avoir fourni une analyse critique du texte, nous souhaitons cependant en explorer plus avant les fondements conceptuels.

2 Notre approche est néo-classique. C'est-à-dire qu'elle est enracinée dans les théories rhétoriques de l'Antiquité. C'est cette même source qui a produit le néo-aristotélisme, «le mode de critique rhétorique dominant de ce siècle aux Etats-Unis » (Black 1965 : 27). Notre analyse critique partage la plupart des présupposés de ce courant traditionnel, avec cependant une différence essentielle. Considérant «les discours rhétoriques comme des actes de communication distincts dans des contextes spécifiques» (Black 1965: 35), le néo-aristotélisme traite du particulier. Cette orientation implique le rejet des critères formels, et contraint presque le chercheur à se fonder sur le critère de l'effet empirique. Nous abordons, quant à nous, le discours de Lincoln dans le cadre d'une conception classique des genres oratoires. A l'opposé du 
nominalisme implicite du néo-aristotélisme, la théorie des genres autorise une conception abstraite de l'auditoire et des situations rhétoriques. Par conséquent, elle permet de fonder le jugement critique sur la prise en compte des dimensions formelles du discours.

Notre but consiste ici à explorer dans quelle mesure la théorie des genres pourrait permettre de remédier à certains défauts inhérents au mode de critique néoaristotélicien. Nous commencerons tout d'abord par faire référence au développement du néo-aristotélisme, en commentant son rejet des distinctions génériques et en montrant les limites qui en découlent. Nous suggérerons ensuite que la théorie des genres peut insuffler une nouvelle vigueur à une analyse critique qui se veut tributaire des modèles classiques. Nous insistons néanmoins sur le caractère provisoire de notre formulation que nous ne considérons pas comme définitive, reconnaissant que notre version du néo-classicisme possède elle-même ses limites.

\section{La critique néo-aristotélicienne}

Bien que le point de vue néo-aristotélicien apparaisse le plus clairement lorsque l'on passe de " The Literary Criticism of Oratory » [ "La critique littéraire de l'art oratoire »] (Wichelns 1972) à A History and Criticism of Oratory [Histoire et critique du discours public américain] (Hochmuth 1955) et à Speech Criticism [La critique du discours oratoire] (Thonssen \& Baird 1948), Wichelns reste l'autorité en ce domaine. Dans son essai qui « définit le modèle » de cette approche « et en détermine l'orientation» (Bryant 1958: 5), la direction qu'il a empruntée est «fermement ancrée dans certaines conceptions aristotéliciennes " (Thonssen \& Baird 1948: 15). La principale en est le caractère primordial de l'auditoire. Bien sûr, Aristote et les autres théoriciens classiques n'établissent pas clairement de système critique, mais

ils en impliquent un. Si on peut dire de l'œuvre littéraire qu'elle a trois références -

l'une à l'univers, l'autre à l'écrivain et la troisième à l'auditoire, la théorie rhétorique grecque, comme les études critiques de la Renaissance qui en dérivent, accorde de loin la plus grande importance à la référence à l'auditoire (Russell 1967 : 141-142).

5 Cherchant à revivifier la perspective rhétorique, Wichelns fait de la référence à l'auditoire le fondement d'une analyse de l'art oratoire. On ne saurait trop souligner l'importance de ce principe; sans lui, l'analyse critique moderne du discours oratoire n'aurait pu se développer. Néanmoins, la détermination de Wichelns à séparer la rhétorique de la littérature ne manque pas de conduire à une conception restreinte de l'auditoire.

Comme le suggère le titre même de son essai, il juge qu'il importe d'établir une distinction entre l'activité littéraire et rhétorique. L'écrivain, affirme-t-il, est libre de suivre sa propre loi (1972 : 56) car la littérature traite de « valeurs permanentes » (ibid. : 57). L'orateur, par contre, est "perpétuellement subordonné à la situation et à l'auditoire " (ibid. : 56). Par conséquent, la critique de l'art oratoire doit considérer le discours comme " un acte de communication destiné à un auditoire spécifique » et se préoccuper de l'effet produit (ibid.: 54). La permanence et l'universalité sont dès lors réservées à la critique littéraire, car lorsqu'un orateur "s'adresse à un auditoire universel », « il ne peut en résulter que confusion » (ibid.: 57). La critique rhétorique trouve donc sa raison d'être dans la spécificité d'une situation particulière, et c'est en 
ces termes qu'elle doit interpréter la fonction de "personnalité », de "preuve ", d'« organisation", de "formulation", ainsi que l'ensemble des thèmes abordés par Wichelns, tous issus de la théorie classique (ibid. : 56-57).

7 Si Wichelns parvient à hisser la critique rhétorique au rang d'activité indépendante, son projet laisse par contre dans l'ombre certains aspects formels de l'héritage classique. Malgré ses nombreux emprunts à Aristote, Wichelns ne dit rien des genres oratoires. Ceci n'est certes pas un hasard, car la théorie des genres est manifestement abstraite ; elle rejette le temps et le lieu comme bases de classification et regroupe les situations historiques en catégories générales. Partant de là, elle soulève des questions sur « la relation entre le groupe et les individus qui le composent, entre l'unité et le nombre, et la nature des universaux » (Welleck \& Warren 1956: 237 ; nous traduisons). Un tel questionnement ne peut s'intégrer au programme de Wichelns car il « sent » le jugement littéraire. Ce n'est qu'en littérature que l'on peut entendre "la voix de l'esprit humain s'adressant aux hommes de tous les âges et de toutes les époques » (Wichelns 1972 : 57), une voix qui transcende les situations particulières.

8 Les néo-aristotéliciens tentent de corriger ce particularisme et d'y échapper. Par exemple, on peut lire qu'une bonne critique « appréhende l'événement dans sa totalité en lui assignant son rang dans l'ensemble de l'entreprise d'énonciation » (Thonssen \& Baird 1956 : 18) et que le discours peut être étudié «comme un produit fini possédant certaines caractéristiques rhétoriques qui se conforment volontiers à des principes ou des règles fixes" (ibid. : 457). On entend parfois aussi évoquer la notion de pierre de touche. Mais assigner son rang à un événement, ou prendre en considération la disposition à se conformer à des principes fixes, implique une certaine idée de permanence ; or, celle-ci est tout simplement absente d'un système tout entier orienté vers les situations spécifiques. Là, le critère adopté est celui de l'effet immédiat, et cet unique critère rend difficile, sinon impossible, de mettre en place des normes artistiques intrinsèques. Le système ne fournit pas de mécanisme logique permettant de relier entre eux des événements en apparence disparates.

Dans la mesure où Aristote parle des genres oratoires, il semble qu'il pourrait offrir aux néo-aristotéliciens une source permettant de générer une analyse formelle; mais les tenants de ce courant, prisonniers du nominalisme rigoureux de Wichelns, ne sont pas à même de l'exploiter. Ils peuvent se référer aux genres, mais non faire usage des distinctions instituées par ceux-ci ; limitant ou divisant les analyses, ils ne les dominent pas ${ }^{1}$. Ironiquement, les néo-aristotéliciens pèchent envers les genres lors même qu'ils utilisent la taxinomie dont ils relèvent ; les chercheurs tendent à classifier « certaines grandes propriétés regroupées sous les modes et les canons traditionnels », afin de "rendre compte mécaniquement de l'adaptation du discours à un moule préexistant " (Ehninger $1965: 230$ ). Sans doute tout système critique a-t-il recours à certains traits fixés a priori, mais il semble que le moule de la critique traditionnelle soit devenu taxinomique sur un mode purement mécanique. Dépouillée des distinctions génériques, la taxinomie néo-classique brise le lien entre l'appareil critique et les objectifs plus larges du discours rhétorique, détournant ainsi l'analyse critique de la relation considérée comme sa raison d'être même: celle qui s'établit entre l'orateur et l'auditoire. Cherchant un refuge, elle se tourne vers la biographie ou l'histoire, mais ni l'une ni l'autre n'offre de solution adéquate. Le fait que la preuve par les détails historiques ne représente pas une échappatoire valable ressort clairement d'un essai qui représente « le meilleur de la tradition néo-aristotélicienne » (Scott \& Brock 1972 : 
21) : l'analyse que fait Marie Hochmuth Nichols du «Premier discours inaugural de Lincoln ».

10 La caractéristique la plus marquante de cet essai est l'accumulation des détails historiques. Evitant les généralisations, Hochmuth Nichols invite le lecteur à participer à l'atmosphère qui caractérisait la situation en rendant compte des détails dont elle était composée. Négligeant le texte du discours inaugural lui-même, les deux tiers de l'article documentent scrupuleusement (Black 1965: 41) les évènements qui l'ont immédiatement précédé ou suivi. Cette procédure peut servir d'adjuvant, mais l'épreuve finale consiste à voir si l'analyste utilise l'accumulation de ces détails de manière à contribuer à la compréhension et à l'évaluation de la transaction rhétorique. Hochmuth Nichols fait face à ce devoir dans le dernier tiers de l'essai, mais elle ne parvient pas à surmonter les limites inhérentes au programme proposé par Wichelns. Fidèle à ses conceptions, elle évalue le discours "comme un medium distinct des autres, possédant ses méthodes particulières » (1972: 88). Dans cette perspective, les éléments auxquels elle se réfère en particulier sont «le choix des sujets abordés par Lincoln, la manière dont il les organise, son style et son mode d'expression » (ibid. : 90). Qu'est-ce qui constitue l'unité de ces diverses orientations de recherche? C'est le but du locuteur. Cependant, Hochmuth Nichols met en garde contre l'hypothèse superficielle selon laquelle "l'objectif de Lincoln [serait] facilement discernable à travers le discours lui-même ", et rejette implicitement les considérations génériques lorsqu'elle se désintéresse du fait qu' « il s'agissait d'une cérémonie inaugurale, avec un rituel assez bien établi » (ibid. : 89). Le genre n'est pas pertinent pour elle parce que les objectifs de Lincoln résultent des expériences « de la nation entre son élection comme Président et le jour de son inauguration » (ibid.). Ces expériences se rapportent au parti, à la nation et à l'homme, et mènent à la conclusion suivante :

Il avait clairement l'intention de profiter de l'occasion de l'inauguration pour proclamer la position du Parti républicain face au Sud, présenter ses réflexions concernant les questions pratiques soulevées par le mouvement de sécession et, globalement, communiquer dans toute la mesure du possible l'assurance de son intégrité personnelle (ibid. : 90).

11 Comparé au caractère spécifique de la documentation historique qui compose la première partie, l'analyse qui suit cette déclaration d'intentions est vague. La critique se limite en grande partie à un résumé. Incluant de nombreuses citations du texte et se livrant à des comparaisons avec les brouillons qui ont précédé la version finale, elle reproduit sans aucun doute le fil de l'argumentation de Lincoln. Cependant, elle laisse au lecteur le soin de sonder les formes et les thèmes de l'argumentation, la nature des preuves, l'interaction entre les parties du discours, et les modes d'appel à l'auditoire. Le résumé laisse dans l'ombre la manière dont Lincoln «est devenu une figure de père affectueux, de conseiller bienveillant générateur d'espoir » (ibid. : 95). De plus l'analyse du style n'examine pas le texte en profondeur; le commentaire ne devient rigoureux et spécifique qu'en termes de référence externe, lorsqu'il se rapporte à la formulation des premiers brouillons. S'il est intéressant d'appréhender le style de Lincoln en prenant en compte le langage qu'il n'utilise pas, il est nécessaire de procéder à une analyse plus serrée de celui qu'il utilise effectivement pour pouvoir soutenir que son style produit « l'image d'un homme au grand cœur, de grande humilité et de grande foi » (ibid. : 99).

Pour éviter tout malentendu, soulignons que notre but n'est pas de diminuer l'importance de cet essai. Nous souhaitons simplement mettre en évidence le fait que son fondement critique est totalement lié au contexte spécifique du discours. Ce lien 
produit, à son tour, une analyse qui se concentre sur les circonstances extérieures au discours plutôt que sur son développement interne. Les limites qui en découlent apparaissent de manière frappante lorsque Hochmuth Nichols tente d'effectuer une évaluation globale du texte.

Sa position est absolument claire: ce Discours inaugural est un chef d'œuvre rhétorique. Mais sur quoi ce jugement est-il fondé ? Ni sur la valeur littéraire du texte ni sur sa prégnance culturelle; ces critères sont explicitement réservés aux critiques littéraires et aux historiens (ibid.: 88). Tout revient donc à l'effet, à la question de savoir si le discours fait bien ce qu'il était supposé faire. Et ce discours particulier était censé énoncer la politique d'un parti, apaiser les craintes des sécessionnistes, et établir l'intégrité personnelle de l'orateur. La réaction du Sud devient donc l'indice principal du succès. Toutefois, au vu de ce critère, le discours a connu l'échec le plus cuisant. Il n'a pas réussi à adoucir les sécessionnistes. En fait, Hochmuth Nichols soutient de manière convaincante qu'après avoir examiné le Discours inaugural, «le Sud ne mit que peu d'espoir dans Lincoln» (ibid. : 85). Mais elle évite la conclusion qui en découle inexorablement. Ignorant l'effet immédiat, elle prétend que « tout critique honnête, se tenant à l'écart des passions de l'époque, doit être bien plutôt en accord avec les observateurs de l'époque pour qui le Discours inaugural remplissait les 'exigences d'une bonne rhétorique'«(ibid.: 95). Cette position est inadmissible: il est parfaitement illogique de mettre l'accent sur la situation spécifique particulière de façon exclusive, puis de demander que l'évaluation finale "se tienne à l'écart des passions de l'époque ".

Le problème que nous venons d'esquisser semble insoluble dans le cadre de la tradition néo-aristotélicienne. Et la remarque de Parrish, selon laquelle dans l'art oratoire «ce n'est pas l'échec qui est un crime, mais le fait de viser bas " (ibid.: 100) ${ }^{2}$ n'offre qu'une faible consolation. Elle ne fait que dissimuler un écart inévitable entre l'allégeance au particulier et le désir d'un cadre de référence plus permanent. Mais si Parrish identifie correctement le besoin de normes abstraites, il ne fournit pas le soubassement nécessaire au formalisme qu'il propose. Nous pensons qu'un tel fondement peut être fourni par la théorie des genres. Pour expliciter cette position, nous devons nous tourner vers les Anciens.

\section{La théorie des genres}

Contrairement à la position de Wichelns, le point de vue dominant de l'ancienne rhétorique n'est pas que les situations sont uniques et particulières, mais qu'elles peuvent être classées selon des catégories générales, des "types» ou "genres». L'influence d'Aristote sur ce point a été décisive. Il décrit trois genres familiers, le délibératif, le judiciaire et l'épidictique, soutenant que « les genres oratoires sont au nombre de trois ; car il y a trois sortes d'auditeurs » (Rhétorique I, 3, 1358b, 36) ${ }^{3}$. Puisque c'est à l'auditeur que "se rapporte la fin » (ibid. I, 3, 1358b, 36), il est l'élément central du système. Par conséquent, Aristote ouvre la voie à l'idée consensuelle selon laquelle "l'aspect important de la situation de discours est la relation orateur-auditoire " (Thonssen \& Baird 1948 : 15). En même temps, cependant, il remarque des similitudes entre certaines sortes d'auditoires et certaines situations discursives et met l'accent sur ces points communs; sa théorie toute entière repose fondamentalement sur une classification abstraite des conditions oratoires. 

genres ; ainsi Cicéron, Quintilien et d'autres auteurs décrivent-ils systématiquement la théorie de l'invention rhétorique en termes de ces trois types de discours. Qui plus est, les genres donnent son sens à l'ensemble de la taxinomie complexe de la rhétorique classique. Les cinq officia et leurs sous-structures détaillées n'ont de sens que dans la mesure où elles aident l'orateur à atteindre son but. Celui-ci, bien sûr, est conditionné par la nature de l'auditoire ; l'orateur, cependant, situe son public par rapport au genre à l'intérieur duquel il remplit sa tâche. Sans les genres, la théorie rhétorique classique perd tout contact avec l'auditoire et sa taxinomie complexe, vidée de sa signification, devient un système privé de but rhétorique. C'est pourquoi nous estimons que tout système critique qui se réclame des modèles classiques doit restituer sa pleine importance à la théorie des genres.

17 L'avantage immédiat de la critique générique est qu'elle permette la création de normes intrinsèques sans perdre de vue l'auditoire. Etabli sur les «attentes de l'auditoire et les exigences de la situation " (Jamieson 1973: 163), le concept de genre suppose que certains types de situation provoquent des besoins et des attentes similaires chez l'auditoire. Le fondement de l'analyse consiste à identifier et catégoriser ces situations ; le chercheur doit mettre au jour les horizons d'attente et les besoins qui assurent la cohésion des auditoires, même si ceux-ci sont éloignés dans le temps et dans l'espace. La description adéquate d'un genre doit par conséquent aboutir à un index général des exigences de l'auditoire; elle situe donc concrètement les problèmes rhétoriques abstraits auxquels l'orateur se trouve confronté et met l'accent sur les normes d'évaluation intrinsèques d'un type particulier de discours.

illustrerons le soubassement générique de notre approche en reprenant notre analyse du Discours de Cooper Union. Il s'agit d'un discours électoral, forme oratoire bien connue des auditoires américains et qui peut être aisément distinguée des autres types de discours publics. Néanmoins, cette forme n'entre pas dans la division tripartite aristotélicienne. L'anomalie devient évidente lorsque nous prenons en considération l'auditoire du discours électoral et son but, composantes de base de la distinction aristotélicienne. L'auditeur d'un discours électoral est le juge d'un événement futur, et il est poussé à accomplir une certaine action (par exemple voter pour un candidat particulier). Cette caractéristique correspond à la fonction de l'auditoire dans le cas du discours délibératif. Le jugement, néanmoins, ne porte pas sur une politique, comme c'est le cas dans le délibératif, mais sur une personne, comme c'est le cas dans l'épidictique. Les objectifs sont également brouillés ; dans le cas du discours délibératif, l'orateur examine «l'utile et le nuisible » d'une ligne de conduite proposée ; l'orateur épidictique doit, quant à lui, "louer ou blâmer " un homme, afin de prouver qu'il est «digne d'honneur ou le contraire» (Rhétorique, I, 3, 1858b, 22-28)4. Or, le but de la campagne électorale est de faire apparaître le candidat comme digne d'éloges et respectable. Néanmoins, prétendre que le discours électoral est une forme de l'épidictique n'a pas de sens, puisque l'objectif de l'orateur est de provoquer une décision, et les auditeurs sont appelés à juger, et non pas simplement à se poser en spectateurs. Aucun des genres traditionnels n'est évidemment entièrement satisfaisant dans ce cas, et il s'ensuit qu'une bonne application de l'approche néo-classique implique la définition d'un genre distinct pour le discours électoral.

Dans cet exemple, la difficulté posée par le système traditionnel paraît être une omission plutôt qu'un défaut inhérent, et il nous semble relativement facile de définir 
ce genre tout en restant cohérent avec les principes aristotéliciens. En nous fondant sur une analyse de l'ethos effectuée par Rosenthal que nous trouvons particulièrement suggestive, nous avons appréhendé le discours électoral comme un exemple de "persuasion personnelle », un discours dont le souci principal est d'orienter l'attitude de l'auditoire envers le candidat (Rosenthal 1966: 114-126). Dans cette optique, la première responsabilité de l'orateur de la campagne électorale sera, par définition, de faire la promotion de sa propre personne en tant qu'individu. Le programme politique et la personne sont tous deux à l'ordre du jour, mais le programme politique apparaît comme subsidiaire par rapport à l'objectif de créer une identification mentale et émotionnelle entre l'orateur et l'auditoire - une exigence qui est inhérente à la situation. L'objectif ultime de l'orateur est de s'insinuer dans les bonnes grâces du public. Traduisant tout ceci dans une terminologie néo-classique, nous pouvons décrire le genre du discours électoral comme une prestation oratoire dans laquelle les auditeurs agissent comme les juges d'un évènement futur, une élection : l'objectif est de produire un jugement sur le candidat, basé sur sa personne et sur la manière dont il traite des problèmes de la cité.

Cette prise en considération des exigences génériques nous a aidés à recentrer notre analyse du Discours de Cooper Union. Dans ce cas spécifique, notre description du genre a suivi Aristote d'assez près. D'autres situations peuvent exiger une adaptation plus radicale du système traditionnel. Il serait certainement déraisonnable de s'attendre à ce que la division tripartite de l'Antiquité englobe toutes les formes de discours public qui sont apparues par la suite. Les genres sont à l'image des institutions (Welleck \& Warren $1956: 226$ ). Ils présentent un certain degré de stabilité à travers les âges, mais ils se développent également, ils changent et tombent en désuétude en réaction à l'évolution de la société. Ils ne doivent donc " pas être considérés comme des formes statiques mais comme des phénomènes évolutifs» (Jamieson 1973: 168). L'analyse critique doit conserver la souplesse nécessaire pour s'adapter aux changements de circonstances. Elle doit savoir que les distinctions génériques ne peuvent faire entrer les textes de force dans des catégories préconçues; sa fonction propre est plutôt de mettre au jour les véritables points communs et les différences entre les formes de discours.

21 La découverte de ces similitudes et de ces dissemblances a pour résultat l'établissement de contraintes génériques, dont l'utilisation s'avère résoudre le problème le plus troublant de la critique néo-aristotélicienne: la tension antinomique entre l'attachement à une situation particulière et le besoin de standards formels d'évaluation. En théorie, dissiper ces tensions peut rendre sa pertinence au néoclassicisme et en restituer l'usage à la critique rhétorique. Nous souhaitons que notre étude du discours de Cooper Union éclaire une partie de ce potentiel. Seule l'analyse elle-même peut témoigner de notre succès ou de notre échec, mais sa genèse mérite un commentaire parce qu'elle aide à expliciter le principe qui sous-tend l'étude et en désigne à la fois les points forts et les limites.

\section{Les usages du néo-classicisme}

La position que nous avons adoptée dans cet essai, comme dans nos autres travaux, s'accorde clairement avec l'appel de Black en faveur d'une "alternative au néoaristotélisme» (Black 1965: 132), et la littérature scientifique indique que notre 
recherche a porté ses fruits. Néanmoins, il semble que cet accord s'est transformé en la recherche d'une "alternative quelle qu'elle soit au néo-aristotélisme ». Or, si le néoclassicisme est devenu mécanique, son rejet arbitraire semble une réaction déraisonnable. Renoncer complètement à la théorie traditionnelle est une démarche douteuse, et l'examen des sources originales nous a convaincu que le retour aux distinctions génériques offrait une alternative plus féconde. Dans une large mesure, la théorie des genres prévient l'argument selon lequel la taxinomie néo-classique limite le chercheur à un ensemble statique de catégories et fait dévier le véritable but de la critique. Elle ancre spécifiquement le discours dans le contexte d'autres discours et dans les exigences de l'auditoire, et met en échec l'accusation selon laquelle l'orientation néo-classique forcerait l'analyste à ignorer « les effets qu'ont l'auditoire, la situation et les autres discours contemporains sur le comportement de l'orateur » (Munshaw 1973 : 30).

23 Le système maintient des traits schématiques, mais cela semble un avantage plutôt qu'un désavantage. Si le travail du critique n'est pas purement capricieux et subjectif, certains schémas se doivent de contrôler l'analyse. Le critique peut se concentrer sur des métaphores archétypales, des composantes dramatiques, des analogies tirées de l'anthropologie et de la mythologie, mais dans tous les cas, des normes pour dégager et sérier les matériaux sont nécessaires. Dans cette optique, la taxinomie néo-classique n'est que l'un des nombreux schémas disponibles et en tant que telle, elle présente l'immense avantage de chercher à traiter dans ses propres termes l'ensemble de la transaction rhétorique. Dernièrement, les analyses les plus marquantes et les plus édifiantes ont eu tendance à adopter un biais non traditionnel, mais nombre d'entre elles ont tendance à isoler et mettre en valeur un élément ou un concept unique. Une approche néo-classique complète se focalise au contraire sur le processus rhétorique et le fractionne en un ensemble simple mais exhaustif de constituants. Hors contexte, ces catégories pourraient présenter un caractère despotique, mais tout autre schéma peut pareillement devenir aliénant, et la théorie des genres aide à déjouer l'établissement d'une tyrannie néo-classique. Maintenant la taxinomie dans ses limites propres, elle fixe l'attention là où elle doit l'être, c'est-à-dire sur la relation orateur-auditoire, et le dispositif ainsi produit paraît constituer un excellent filtre pour l'analyse des discours particuliers. Examinant le Discours de Cooper Union, par exemple, nous ne nous sommes pas sentis prisonniers d'une orthodoxie compliquée et abrutissante, ni enfermés dans des préoccupations secondaires. Nous avons au contraire senti que le système nous forçait à nous attaquer au discours en lui-même, et à faire des choix ${ }^{5}$. La question de savoir si nous avons fait les bons choix est secondaire par rapport au fait que nous ne nous sommes pas sentis enfermés dans des limites arbitraires propres à affaiblir l'analyse.

Le système, bien sûr, a ses limites. La théorie des genres suppose des types, et l'analyste utilise généralement le type pour explorer un exemple unique. Les comparaisons sont certes possibles; de même, l'analyse pourra porter sur une série d'un même genre; cependant, le néo-classicisme convient à l'analyse d'un discours spécifique plus qu'à tout autre chose. Le système ne peut certes traiter des phénomènes de "piquetage, slogans, éloge, chant, manifestation et signaux » et faits de communication similaires (Bitzer \& Black 1971 : 225), mais cette limite ne fait que circonscrire le champ dans lequel œuvre le néo-classicisme. Par contre, l'argument selon lequel la critique néo- 
classique souffre d'un biais rationaliste pose un problème plus grave (voir Black 1965 : 91-131 et Campbell $1970:$ 97-108).

Dans une perspective générique, ce biais est institutionnel. Les genres rhétoriques sont fondés sur des pratiques établies, et la théorie des genres présume l'existence de conventions stables qui régulent la communication publique. Misant sur ce consensus public, la théorie des genres propose une prise en compte cohérente et donc « rationnelle » de l'argumentation rhétorique. Cette approche présente des avantages dans des circonstances normales, mais soulève des problèmes lorsqu'elles cessent de l'être, en période de changements rapides. Ici réside la limite la plus importante du système critique que nous avons entrepris de défendre.

Ce système marche nécessairement mieux dans des situations où l'étendue du désaccord est limitée, lorsqu'il existe un large consensus sur ce qui peut être discuté et sur la manière de le faire. Par conséquent, même un observateur aussi sympathisant que Booth doit reconnaître que "la tradition aristotélicienne convient mieux à l'analyse de la cohérence de [...] la rhétorique, du point de vue de quelqu'un qui se situe, au moins dans une certaine mesure, à l'intérieur» (Booth 1971: 97). Notre proposition porte donc sur une rhétorique vue de l'intérieur. Pourtant, chacun est conscient de la rhétorique de celui qui se situe à l'extérieur du système, « la rhétorique de 'conversion', de transformation, la rhétorique ayant pour effet, déclaré ou non, de renverser les liens personnels et de modifier totalement les allégeances » (Booth 1971 : 102). Cette rhétorique radicale peut attaquer explicitement les conventions et les institutions stables d'une société donnée ; de plus, elle est susceptible d'apparaître sous une forme qui défie les normes de discours établies, car les tentatives de subvertir l'« establishment » impliquent presque toujours une tentative de subvertir le langage.

Dans de telles situations, la théorie des genres vacille. Le système, fondé sur une présomption de stabilité, ne possède pas de mécanisme permettant d'expliquer des changements dramatiques d'idéologie ou de style de type idiosyncrasique. La critique pourra s'adapter avec le temps, mais une flexibilité à long terme ne résout pas le problème lorsqu'on s'efforce d'expliquer une rhétorique radicale in situ. Pis encore, le choix d'adhérer à une typologie conventionnelle peut emprisonner l'analyste dans le système institutionnel que les formes rhétoriques émergentes cherchent précisément à détruire. Leur analyse condamnera les tenants $d u$ changement parce que leur rhétorique ne se conforme pas aux attentes conventionnelles, et cette rhétorique peut alors donner lieu à méprise. Néanmoins, même cette limite peut s'avérer d'une certaine manière productive, si elle est évaluée comme il convient.

Une période de changement rapide et radicale peut nécessiter, plus que toute autre, des points de référence stables. Il est certes toujours hasardeux d'évaluer de nouveaux types de discours en fonction des anciens, mais il n'y a pas de raison pour que les genres existants ne puissent servir de pierre de touche pour la compréhension de nouvelles formes. En d'autres termes, la critique rhétorique pourrait «prendre pour point de départ l'idée selon laquelle l'étude des changements historiques pourrait tirer un large bénéfice d'une vue plus claire de ce qui est en train de changer que celle que nous possédons à présent » (Hernadi 1972). Les bases d'un fondement critique adéquat pour rendre compte des brusques changements d'idéologie sont encore à établir. 


\section{Conclusion} proposons tout simplement d'utiliser la théorie des genres comme moyen d'introduire du formalisme dans la critique néo-classique. Cadrant bien avec la tradition, cette théorie ne demande pas d'abandonner la centralité de la notion d'auditoire. En fait, la théorie des genres agit comme un correctif précisément parce qu'elle permet une conception plus large de l'auditoire que celle qu'a autorisée la conception néoaristotélicienne. Si l'analyse critique peut identifier des situations qui créent généralement des demandes similaires de la part de l'auditoire, elle devient capable de définir des principes applicables à une large variété de discours. Bien que ces normes reposent sur une analyse de l'auditoire, celui-ci est conçu en termes génériques, et les généralisations qui s'ensuivent peuvent offrir des principes formels de base pour évaluer les discours d'un type particulier. Ces normes ne possèdent peut-être pas les caractéristiques formelles de certains genres littéraires, mais elles permettent de pénétrer dans l'espace d'un discours donné et d'orienter la réaction critique.

reste beaucoup à faire, bien sûr. Notre analyse du genre électoral est encore limitée et expérimentale. Dans le cadre de la taxinomie néo-classique, nous avons établi une définition à priori de l'objectif du discours électoral, et avons pu ainsi construire des normes rhétoriques d'évaluation très larges utilisables pour un cas particulier. Il est nécessaire d'étudier des exemples supplémentaires si l'on veut pouvoir décrire adéquatement le genre et dégager des contraintes génériques plus spécifiques. De façon générale, nous n'avons pas examiné en détail la théorie des genres, ni tenté d'établir une classification systématique des genres rhétoriques modernes.

Fort heureusement, les résultats de la recherche appliquée ont confirmés ces orientations (voir Black 1965: 132-177, Hill 1972 : 373-386, Ware et Linkugel 1973 : 273-283 ; Jamieson 1973). La plus grande partie de l'effort a été mené de façon fragmentaire, mais ceci, après tout, peut s'avérer être le chemin le plus satisfaisant. Les recherches modernes en analyse rhétorique commencent généralement par une prise de position théorique avant de passer à son application, alors qu'en écrivant ces deux articles, nous avons été amenés à aborder la question dans la perspective opposée. L'expérience a été édifiante : elle mérite peut-être d'être généralisée. Il se peut que le progrès de la critique rhétorique repose davantage sur la capacité des analystes à induire des principes à partir d'études concrètes que sur le souci des questions abstraites.

\section{BIBLIOGRAPHIE}

Aristote. 1967. Rhétorique, 3 vol. Trad. Médéric Dufour (Paris : Les Belles Lettres)

Bitzer, Loyd F. \& Edwin Black (eds). 1971. The Prospects of Rhetoric (Englewood Cliffs, NJ : PrenticeHall) 
Black, Edwin. 1965. Rhetorical Criticism (New York : Macmillan)

Bryant, Donald C. (ed.) 1958. The Rhetorical Idiom (Ithaca, New York : Cornell University. Press)

Booth, Wayne C. 1971. « The Scope of Rhetoric Today: A Polemical Excursion », Bitzer \& Black (eds). The Prospects of Rhetoric (Englewood Cliffs, NJ : Prentice-Hall)

Campbell, Karlyn K. 1970. « The Ontological Foundations of Rhetorical Theory », Philosophy and rhetoric 3, 97-108

Ehninger, Douglas. 1965. «Rhetoric and the Critic », Western Speech 29, 227-231

Hernadi, Paul. 1972. Beyond Genre (Ithaca, New-York : Cornell Univ. Press)

Hill, Forbes I. 1972. « Conventional Wisdom-Traditional Form-the President's message of

November 3, $1969 »$, Quarterly Journal of Speech 58, 373-386

Hochmuth, Marie Kathryn (ed.) 1955. A History and Criticism of American Public Address (New-York : McGraw-Hill) [Brigance, William Norwood, 1943]

Hochmuth Nichols, Marie. 1972. « Lincolns' First Inaugural », Scott, Robert L. \& Bernard L. Brock. Methods of Rhetorical Criticism (New-York : Harper and Row), 60-100

Jamieson, Kathleen M. Hall. 1973. « Generic Constraints and the Rhetorical situation », Philosophy and Rhetoric 6, 162-170

Jebb, R., C. 1962 [1876]. The Attic Orators (New York : Russell \& Russell)

Parrish, Wayland Maxfield. 1954. « The Study of Speeches », Parrish, Wayland Maxfield \& Mary K. Hochmuth (eds). American Speeches (New-York : David McKay)

Munshaw, Joe A. 1973. «The Structures of History. Dividing Phenomena for Rhetorical Understanding ", Central States Speech Journal 24, 29-42

Rosenthal, Paul, I. 1966. « The Concept of Ethos and the Structure of Persuasion », Speech

Monographs 33, 114-126

Russell, D. A. 1967. « Rhetoric and Criticism ». Greece and Rome, $2^{\text {nd }}$ Ser., 14, 130-144

Thonssen, Lester \& Baird, A. Craig. 1948. Speech Criticism (New York : Ronald Press)

Ware, B. L. \& Wil A. Linkugel. 1973. «They Spoke in Defense of Themselves: On the Generic Criticism of Apologia », Quarterly Journal of Speech 59, 273-283

Welleck, René \& Austin Warren. 1956. Theory of Literature (New York : Harcourt, Brace \& World) Wichelns, Herbert A. 1972. « The Literary Criticism of Oratory », Scott, Robert L. \& Bernard L. Brock. Methods of Rhetorical Criticism (New York : Harper and Row), 27-60

\section{NOTES}

1. Même une lecture superficielle de A History and Criticism of American Public Address montre que les genres n'y sont pas l'objet d'une étude systématique.

2. Parrish (1954: 12). Voir Black (1965 : 61-75).

3. Pour un commentaire sur le formalisme général des arts oratoires antiques, voir Jebb (1962).

4. La traduction française est différente : pour les orateurs « qui louent et blâment, les fins sont le beau et le laid; à quoi ils ramènent, eux aussi, tout le reste » (Note de la traductrice).

5. Dans notre approche, nous avons délibérément supprimé les détails de l'analyse. Par exemple, nous avons ignoré les complications de l'argumentation de la première partie parce qu'elles 
auraient été fastidieuses pour le lecteur et auraient fait apparaître le discours comme plus formel qu'il ne l'est en réalité. Nous avons de même passé d'autres éléments sous silence. Par exemple, nous n'avons pas étudié l'utilisation par Lincoln de la question rhétorique, sauf indirectement, ni $\mathrm{du}$ refrain "Nos pères». Nous aurions pu rapporter ce dernier à chaque segment du débat implicite avec le Sud, et prendre en considération sa valeur d'écho religieux chez un auditoire parfaitement familier de la prière du Notre Père. Nous ne prétendons pas que ces facettes du discours ne seraient pas apparues à un analyste usant d'une approche différente. Nous insistons, cependant, sur le fait que le genre et la taxinomie les ont portées à notre attention. Le fait que nous ne les ayons pas traitées indique nos choix plutôt qu'une quelconque déficience de notre approche.

\section{INDEX}

Mots-clés : approche néo-classique, critique néo-aristotélicienne, discours électoral, genre rhétorique, Lincoln (Abraham)

Keywords : campaign oration, Lincoln (Abraham), neo-Aristotelian criticism, neo-classical approach, rhetorical genre 